\title{
Size of CA1-Evoked Synaptic Potentials Is Related to Theta Rhythm Phase in Rat Hippocampus
}

\author{
BRADLEY P. WYBLE, ${ }^{1}$ CHRISTIANE LINSTER, ${ }^{1,2}$ AND MICHAEL E. HASSELMO ${ }^{2}$ \\ ${ }^{1}$ Department of Psychology, Harvard University, Cambridge 02138; and ${ }^{2}$ Department of Psychology, Boston University, \\ Boston, Massachusetts 02215
}

\begin{abstract}
Wyble, Bradley P., Christiane Linster, and Michael E. Hasselmo. Size of CA1-evoked synaptic potentials is related to theta rhythm phase in rat hippocampus. J. Neurophysiol. 83: 2138-2144, 2000. Cholinergic and GABAergic neurons projecting to the hippocampus fire with specific phase relations to theta rhythm oscillations in the electroencephalogram (EEG). To determine if this phasic input has an impact on synaptic transmission within the hippocampus, we recorded evoked population excitatory postsynaptic potential (EPSPs) during different phases of theta rhythm by using techniques similar to those described in Rudell and Fox. Synaptic potentials elicited by stimulation of region $\mathrm{CA} 3$ of the contralateral hippocampus were recorded in region $\mathrm{CA} 1$ and CA3. In these experiments, the initial slope of evoked potentials showed a change in magnitude during different phases of the theta rhythm recorded in the dentate fissure, with individual trials showing an average of $9.5 \%$ change in slope of potentials, and the average across all experiments showing a change of $7.8 \%$. Evoked potentials were maximal $18^{\circ}$ after the positive peak of the dentate fissure theta EEG. These potentials were also smaller by $18.2 \%$ during theta as opposed to non-theta states. Phasic changes in modulation of synaptic transmission could contribute to phase precession of hippocampal place cells and could enhance storage of new sequences of activity as demonstrated by computational models.
\end{abstract}

\section{N T R O D U C T I O N}

Electroencephalographic (EEG) recordings from the hippocampus of rodents commonly reveal a large amplitude oscillation in the theta frequency range $(3-12 \mathrm{~Hz})$ during active sniffing, exploration, movement, and REM sleep (Bland 1986; Green and Arduini 1954). This theta rhythm shows a remarkable coherence across much of the septo-temporal extent of the hippocampus (Buzsaki et al. 1990) and correlates with theta in the entorhinal cortex (Alonso and Garcia Austt 1987). Accordingly, a large amount of work has been directed at determining the causes and effects of this rhythm. Theta usually appears to be driven by neurons of the medial septum/ventral limb of the diagonal band (MS/VDB) complex. The MS/VDB contains cholinergic and GABAergic neurons that project to the hippocampus (Amaral and Kurz 1985). These neurons fire at theta frequency and are thought to drive both the phase and frequency of hippocampal theta (Brazhnik and Fox 1997; Stewart and Fox 1990; Vertes and Kocsis 1997).

In addition to the prominent EEG signal that is the hallmark of theta, a variety of cellular properties have been found to vary with theta phase. These include changes in firing rate (Buzsaki

The costs of publication of this article were defrayed in part by the payment of page charges. The article must therefore be hereby marked "advertisement" in accordance with 18 U.S.C. Section 1734 solely to indicate this fact. et al. 1983; Fox et al. 1986), excitability (as measured by population spike amplitude (Rudell and Fox 1980; Rudell et al. 1984)], membrane potential (Fox 1989; Kamondi et al. 1998; Leung and Yim 1986; Núñez et al. 1987), and membrane resistance (Fox 1989). Although it is difficult to determine decisively which of these effects are direct results of phasic changes in extracellular acetylcholine or GABA, it is possible to catalog these changes with the intent of achieving a complete model of hippocampal activity with regard to the theta rhythm.

Here we report work in urethan anesthetized rats which exhibit prominent hippocampal theta oscillations either spontaneously or when given a tail pinch. The slope of evoked synaptic potentials caused by CA3 or CA 1 stimulation were recorded in CA1 simultaneously with extracellular theta at the hippocampal fissure to determine if the size of these potentials varied with the phase of theta. These potentials were adjusted to be devoid of contamination by population spikes which could distort the slope measurement. This measurement is distinct from the aforementioned papers in that it uses the slope of excitatory postsynaptic current (EPSPs) untainted by population spikes. It allows one to measure the strength of synaptic transmission rather than the firing of neurons which does not always co-vary with transmission (Green et al. 1990).

This data, published as a preliminary report (Wyble et al. 1997), has proven useful to computational models that examine the function of the theta rhythm (Sohal and Hasselmo 1998a,b; Wallenstein and Hasselmo 1998).

\section{METHODS}

\section{Surgical preparation}

Male Sprague-Dawley rats were given IP injections of urethan anesthesia $(0.9 \mathrm{~g} / \mathrm{kg})$ and periodic administrations of metofane by inhalation until a deep level of anesthesia was reached, as confirmed by lack of response to a footpad pinch of the hind limbs. Body temperature was maintained through the use of a heating pad at $37^{\circ} \mathrm{C}$. The skull was exposed and holes were drilled at stereotaxic coordinates described below. Coordinates were in accord with Paxinos and Watson (1986).

\section{Stimulation and recording electrodes}

Recording electrodes and bipolar stimulating electrodes were fashioned from stainless steel wires coated in Formvar, 0.004 in. diam, cut square. The recorded signals were passed through Grass P15 preamplifiers and a Neuralynx amplifier before being passed to a data translation A/D board for storage by Experimenter's Workbench software from DataWave. Amplification level for all signals was set at 


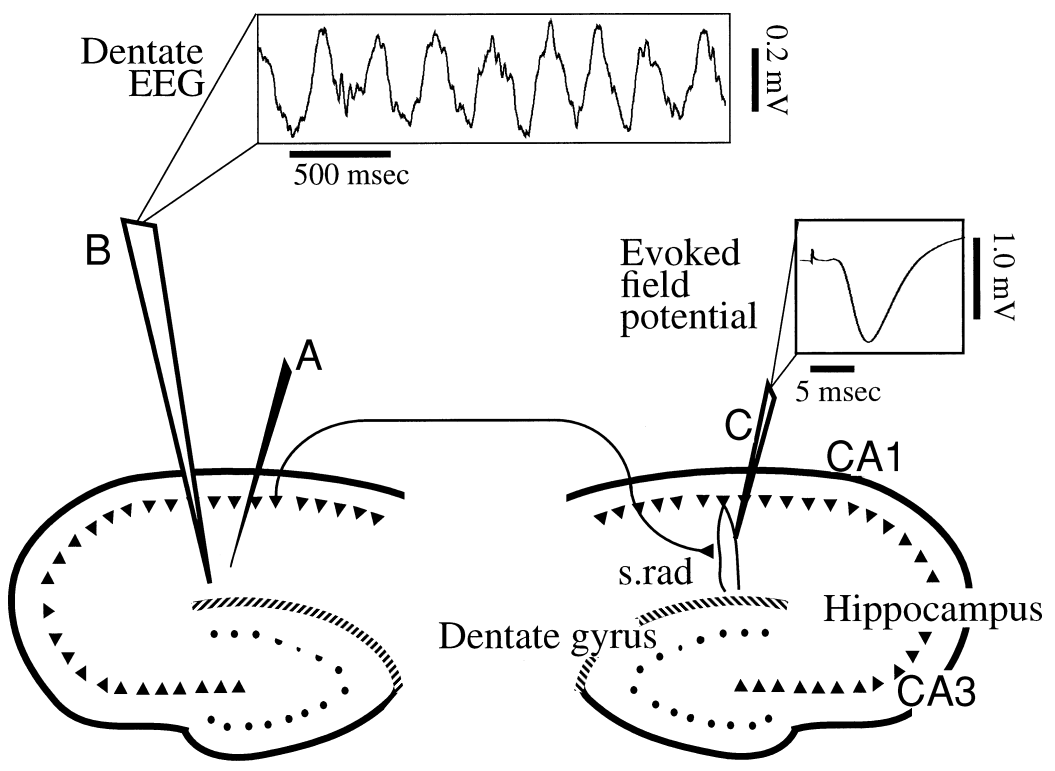

FIG. 1. Schematic diagram of experimental design. Stimulation through electrode A caused evoked synaptic potentials in the contralateral hippocampus, which were recorded with electrode $C$. Electrode $B$ was positioned just above the hippocampal fissure on the stimulation side to record a large amplitude theta rhythm in the electroencephalogram (EEG).

2,000. Electrical stimulation was generated by a Grass PSIU6 stimulus isolation unit driven by a Grass S88 stimulator.

Placement of stimulation and recording electrodes is summarized in Fig. 1. To record theta rhythm oscillations in the EEG, a recording electrode was lowered into the left dentate fissure $(-5 \mathrm{~mm}$ from Bregma, 2.5-mm lateral, 3-mm deep). To record evoked synaptic potentials, a recording electrode was lowered into the right CA1/CA3 region ( $-3 \mathrm{~mm}$ from Bregma, 2-mm lateral, $\sim 2.5-3.5 \mathrm{deep}$ ). For stimulation of evoked synaptic potentials through activation of contralateral projections, a stimulating electrode was lowered into the left CA1/CA3 region ( $-3 \mathrm{~mm}$ from Bregma, 2-mm lateral, $\sim 2.5-3 \mathrm{~mm}$ deep).

Vertical placement of the dentate fissure electrode was confirmed by simultaneously recording theta through it and the contralateral recording electrode. The dentate electrode was then lowered until an abrupt phase reversal occurred by using the stationary phase of the contralateral electrode as a reference and a very large amplitude theta rhythm oscillation could be recorded (Rudell and Fox 1984). This electrode was adjusted until the theta rhythm it recorded was of maximum amplitude and was antiphasically related to the CA1 theta recorded in the contralateral stratum oriens or pyramidale, as well as could be determined by using a two-channel oscilloscope. Vertical placement of the CA1/CA3 stimulating and recording electrodes were established by examining potentials evoked by stimulation. The electrode depths were adjusted until a maximal, negative evoked potential with no population spike contamination was found within the stereotaxic limits of the hippocampus. The initial slope of these negative potentials was presumed to result from monosynaptic EPSPs generated by synaptic potentials on CA1 dendrites from contralateral CA3 cells. Potentials varied in their peak latency between 9 and $13 \mathrm{~ms}$, with most having a peak latency of about $12 \mathrm{~ms}$. Occasionally the dentate electrode, ipsilateral to stimulation, picked up similar potentials from the stratum radiatum just above it. These potentials were included as three trial sets for two animals and were similar in all respects to data from the contralateral side.

\section{Experimental protocol}

After surgery, the anesthesia level was reduced by withdrawal of metofane and monitored by periodic checking of the hindlimb reflex to a footpad pinch. In this state, theta rhythm could be elicited through a tail pinch, and in some cases, occurred spontaneously.

For each individual trial, $3 \mathrm{~s}$ of EEG data from the dentate fissure electrode were recorded. After a delay of $44 \mathrm{~ms}$, a brief electrical stimulation pulse $(0.03 \mathrm{~ms}$ at $100-200 \mu \mathrm{A})$ was delivered to the stimulation electrode and the resultant potential was recorded on the CA1 recording electrode. Six hundred such recordings were collected for a single trial set. Within each rat 1-4 complete trial sets were recorded, resulting in 600-2,400 trials per animal. During a trial set, periods of non-theta were interspersed with theta by varying tail pinch stimulation. Because theta frequency was not correlated with stimulation frequency, this protocol insured a relatively random and homogeneous distribution of the phases of theta at which stimulation occurred.

\section{Histology}

After each experiment, the animals were lesioned at each electrode location and perfused with a solution containing 25 parts $10 \%$ buffered formalin, 1 part glacial acetic acid, and $10 \mathrm{~g}$ of potassium ferrocyanide. This solution causes a Prussian blue reaction, which marks blue the location of the iron particles deposited by an electrode lesion. The brains were then removed and stored in a $10 \%$ buffered formalin solution for about 1-2 wk. After this time, the brains were then sliced, mounted, and stained with neutral red to determine precise electrode locations.

\section{Data analysis}

THETA VERSUS NON-THETA COMPARISON. The first measurement examined the change in evoked potential slope between those potentials preceded by theta EEG and those preceded by non-theta EEG. To accomplish this, the $3 \mathrm{~s}$ of EEG recorded prior to each stimulation were subjected to a fast Fourier transform (FFT). The power spectrum was broken into theta band (usually $3-4 \mathrm{~Hz}$ with some variation between animals) and non-theta band components (the frequencies between 1-10 Hz not including the theta band). Theta trials for this part of the analysis were those in which theta band activity comprised at least $75 \%$ of the total power (between $1-10 \mathrm{~Hz}$ ), whereas non-theta trials were those in which theta power was $<25 \%$ of the total power. An example of a segment of theta can be seen in Fig. 1. This threshold was set to be conservative such that records with faint or intermittent theta were discarded. To eliminate trials with contamination from other fiber tracts, all sets of trials which had theta suppression significant at $P=0.01$ or less in a two-tailed $t$-test were removed from the analysis. This eliminated 4 out of 26 sets of trials. The bins from the 22 remaining trial sets from nine rats were subjected to further analysis. 
CORRELATION OF THETA PHASE WITH EVOKED POTENTIAL SLOPE. The trial sets not discarded during the previous analysis (22 of 26) were then analyzed to compute the effect of theta phase on evoked potential slope. This analysis involved sorting each trial of a 600 trial set recorded within a rat by the phase of theta at which stimulation occurred and then analyzing the evoked potentials as a function of this phase. The following steps were performed for each trial within a trial set.

1) Check overall theta coherence. The EEG record at the beginning of a trial was examined for theta coherence to insure that theta was robust for the entire 3-s recording as described above. Only trials in which theta band activity comprised at least $50 \%$ of the total power (between 1-10 Hz) were considered for this analysis.

2) Check coherence of last theta cycle. The last cycle of theta before stimulation was cross correlated with different sine waves to ensure that it was possible to determine its phase accurately. Trials were only accepted for which the largest correlation value exceeded $r=0.75$.

3) Determine phase of stimulation. The phase of the most highly correlated sine wave was considered as the phase of stimulation after correcting for the 49-ms delay between the end of theta measurement and the occurrence of the evoked potential.

4) Add potential to appropriate bin. For data analysis the theta cycle was divided into 10 bins, each subtending $36^{\circ}$ of the entire theta period. The first bin corresponded most closely to $18^{\circ}$ after the positive peak of the theta cycle. Each evoked potential was summated with one of these bins, according to the phase of theta at which it occurred. Individual potentials were also indexed by phase to allow derivation of statistical significance.

5) Compute average and standard deviation of slopes for all potentials within each bin. Slope measurement was made between 40 and $60 \%$ of the initial negative portion of each evoked potential. An approximation of the slope of local theta was also computed from the baseline EEG immediately before the stimulus artifact for each stimulation. This local theta slope was subtracted from the slope of the individual evoked potential to eliminate this artifact as a possible source of the effect. The theta rhythm was slow enough relative to the duration of the evoked potentials that the local theta slope did not change appreciably between the time when it was measured and the time when the slope of the negative potential was measured. For each trial set, slope measurements were then normalized to the mean slope of the theta records within that trial set. This allowed comparison of slope measurements across animals. The slopes of individual trials were also computed and normalized for later statistical computation.

6) Construct modulation curve from slope. These slopes were then used to construct a modulation curve for a trial set. This curve yielded a phase of maximal and minimal slope as well as an effect size. These values included the aforementioned local theta subtraction.

After the phase and slope of each evoked potential were determined, data points across all rats were normalized to indicate percent suppression and then combined into an overall average data set which yielded a modulation curve for the entire data set. By combining data this way, trial sets with a low yield of theta trials were given proportionately less weight, and statistics for the entire data set were easily computed. Effect size for the averaged data set over the nine rats was calculated by subtracting the smallest bin from the largest bin. For individual trial sets, the effect size was computed by subtracting the average of the two largest consecutive bins from the two smallest consecutive bins to compensate for the extra variance of the smaller amount of data in each set.

\section{Statistics}

All statistics were performed by using MATLAB version 5.1 (published by The Mathworks) for Linux with the statistics toolbox. This package allowed computation of 1 and 2 factor analyses of variance (ANOVAs) and $t$-tests.

\section{Histological validity}

All experiments included in this analysis had electrode locations determined by successful histological preparation of the hippocampal structures. Contralateral stimulation electrodes were all located in stratum radiatum of CA1 or in the pyramidal cell layer of CA3. Stimulation in stratum radiatum antidromically fired CA3 pyramidal cells which then caused an excitatory potential in stratum radiatum of the contralateral CA1 region. Stratum pyramidale stimulation caused direct activation of CA3 pyramidal cells which caused the same type of potential. Recording electrodes were generally located in stratum radiatum of region CA1. Separation of results for different stimulation locations will be discussed within the results.

\section{RE S U L T S}

\section{Electrode locations}

Stimulation locations were either stratum radiatum of CA1, stratum lacunosum moleculare of CA1, or stratum pyramidale of CA3. Figure 2 illustrates stimulation and recording sites for each animal. Comparison of the amount of theta suppression found in potentials elicited by CA3 pyramidale and CA1 stimulation indicated a slight difference that was not significant on a two-tailed $t$-test $(P=0.083)$. A two-factor ANOVA indicated no significant interaction between the theta phase modulation and stimulation location $[P=0.17, F(1,9,9,3860)=$ 1.424]. Therefore the data from these two sites, which were not significantly different in either measurement, were lumped together to simplify further analysis.
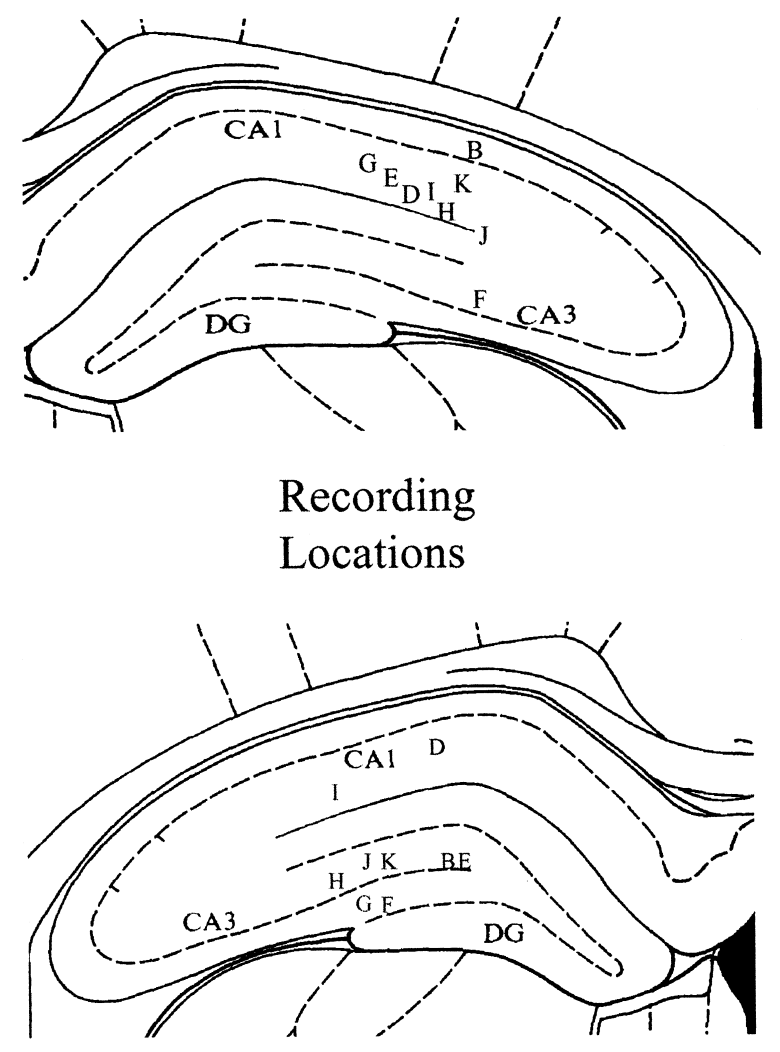

\section{Stimulation Locations}

FIG. 2. Histology of experiments from each of 9 rats. Each letter indicates location of a recording and a stimulating electrode within a given rat. 


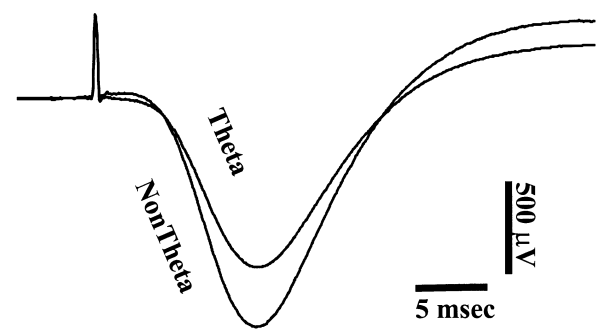

FIG. 3. Evoked potentials recorded during theta and non-theta in 1 rat. Note the obvious suppression of potentials recorded during theta.

\section{Theta versus non-theta}

Of the 26 trials, 22 showed a significant suppression of evoked potential slope during theta relative to non-theta, as summarized in Fig. 3. The average difference between theta and non-theta potentials over all 26 trials was $18.2 \% \pm 0.5 \%$ and was significant at $P<0.0001$ on a two-tailed $t$-test.

\section{Phasic modulation of initial slope of evoked potentials}

Phasic modulation of the slope of excitatory potentials was found within each individual trial set as well as within the averaged data set collected from all nine rats in the analysis. Examples of the average evoked potential obtained at 90 and $198^{\circ}$ after the positive peak of dentate fissure theta from a single trial set is shown in Fig. 4. Over the course of a theta cycle the maximal change in slope of negative going potentials was $7.8 \%$ when averaged over the entire data set. An ANOVA comparing all 10 bins showed their means to be significantly different at a $P<0.00001[F(9,7295)=13.4]$. Figure 5 illustrates the relative size of evoked potentials at different phases of theta for the entire data set. The largest potentials occurred $18^{\circ}$ after the positive peak of the dentate fissure theta EEG (the negative peak of local CA1 theta) and the smallest potentials occurred at $198^{\circ}$ after the positive peak of dentate fissure theta (the peak of local CA1 theta).

Individual trial sets exhibited modulation of EPSP slope between 6 and $16 \%$. Individual differences in the phases of the maximal suppression for each rat decreased the size of the suppression when observed over the entire data set. When the effect sizes within each set of trials were averaged, ignoring these differences in phase, the average change was $9.5 \%$. Results derived without corrective subtraction of theta slope were similar to results obtained with the subtraction. The effect size without this correction was a $7.5 \%$ suppression instead of $7.8 \%$ and showed identical bins of maximal and minimal suppression. Comparison of theta phasic changes in evoked potential amplitude with changes in theta phasic evoked potential slope for each trial set yielded correlation values that ranged from 0.59 to 0.97 , with the average over all trial sets being 0.85 . This strong correlation indicates that the evoked potential slope varied synchronously with its amplitude, at least with regard to different phases of theta.

\section{Secondary correlates}

By correlating measurements obtained from averaged trial sets, it was possible to derive additional information about the modulation of evoked potential size in relation to the phase of

\section{Dentate EEG}

Evoked

Field Potential

\section{EPSP Slope} $\%$ of mean

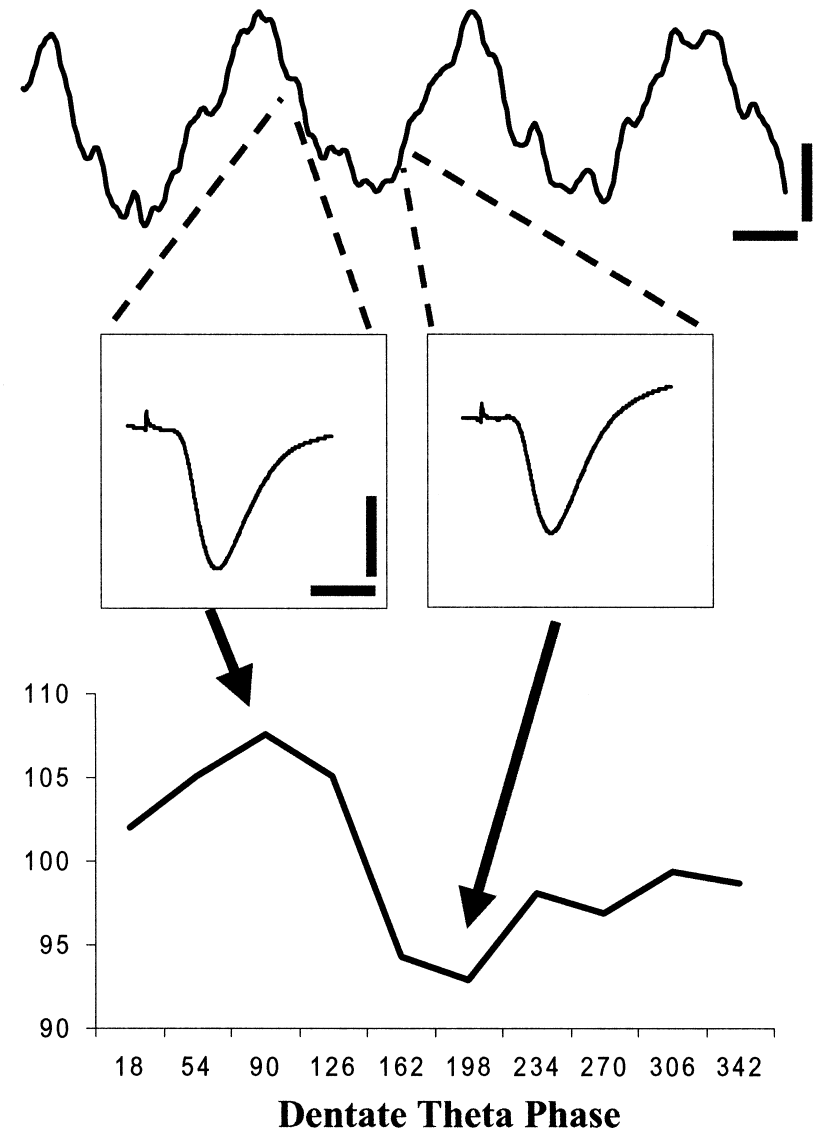

FIG. 4. Examples of average evoked potentials elicited in stratum radiatum of region CA1 in 1 rat during different phases of theta recorded above the dentate fissure. The rising slope of averaged evoked potentials is larger for potentials recorded at $90^{\circ}$ after the positive peak when compared with potentials recorded at $198^{\circ}$ after this peak. The modulation curve shown below the evoked potentials illustrates data from a single set of trials. Over $3 \mathrm{~s}$ of time elapsed between stimulation of evoked potentials during experiments. Placement of evoked potentials on a single theta cycle for illustrative purposes only. Calibration bars: $80 \mathrm{~ms}$ and $200 \mu \mathrm{V}$ (dentate fissure EEG); $5 \mathrm{~ms}$ and $500 \mu \mathrm{V}$ (evoked field potential). 


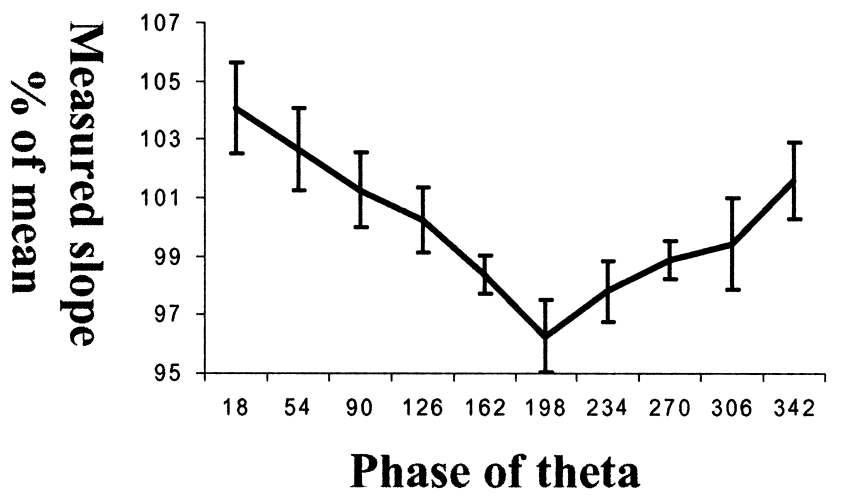

FIG. 5. Change in slope of evoked potentials averaged over the entire data set of 22 trial sets in 9 rats. The slope of the evoked potentials relative to the mean slope is shown for different phases of dentate gyrus theta (broken into 10 bins of $36^{\circ}$ each). Average change in modulation showed an effective suppression of $7.8 \%$, although the average effect size for individual trials was about $9.8 \%$. Error bars, SE (standard deviation divided by square root of $N$ ).

theta, and theta or non-theta state. When the amount of modulation of evoked potential slope within each trial set was compared with the mean size of evoked potential in that set, the modulation of evoked potentials by theta phase seemed to be influenced by the size of the evoked field potential, but not in a consistent manner. In most cases the effect size was larger for smaller stimulus intensities, having average correlation of $r=$ -0.50 . Larger stimulus intensities may have recruited other tracts of fibers in the region, causing volume conducted contamination or direct contamination by synaptic inputs that were not modulated in the same way. Another possibility is that larger stimulus intensities recruited more feedforward inhibition, resulting in rapid shunting of dendritic currents. Similarly, the modulation of evoked potential slope with theta or nontheta condition varied negatively with evoked potential amplitude $(r=-0.53)$. That is to say, larger potentials were less suppressed by theta state than were smaller potentials. This anticorrelation could also be the result of recruitment of contaminating potentials with larger stimulating intensities.

\section{I S C U S S I O N}

\section{Theta phasic parameters in urethanized preparations}

These results demonstrate that the initial slope of monosynaptic evoked potentials within the hippocampus changes at different phases of the theta rhythm. Synaptic potentials are at their smallest just after the negative peak of dentate fissure theta (the positive peak of CA1 theta). The change in EPSPs indicates that synaptic transmission may be changing in addition to previously reported effects of theta on postsynaptic factors influencing population spike generation.

To put these results in the scope of previous work on this topic, neuronal excitability (as measured by population spike amplitude), membrane impedance, and complex spike firing rates are all maximal at approximately the same phase as the largest slope of evoked potentials in this study, namely, near the positive phase of dentate theta (Fox 1989; Fox et al. 1986; Rudell and Fox 1984). Interneuron firing is maximal near the negative peak of dentate theta (Fox et al. 1986), just after the phase of minimal slope of evoked synaptic potentials found here. The membrane potential of both CA1 pyramidal cell dendrites and somata is also known to vary with urethan theta
(Fox 1989; Kamondi et al. 1998; Leung and Yim 1986; Núñez et al. 1987), although some of these reports conflict as regards the phase alignment and magnitude of the effect.

The phenomenon of LTP has also been shown to vary with theta phase as well. Huerta and Lisman (1995) found that stimulation at the peaks of local theta in hippocampal slices tended to cause long-term potentiation (LTP) in CA1 synapses, whereas stimulation at the troughs caused these same synapses to depotentiate. Holscher et al. (1997) found a similar phase preference for LTP induction in urethanized rats by using evoked potentials recorded in CA1 of the dorsal hippocampus. Small stimulation trains at $200 \mathrm{~Hz}$ on the positive phase of local theta (presumably in phase with dentate theta recorded here) induced LTP, whereas a similar train on the negative phase of theta reversed this LTP. Orr et al. (1999) found that potentiation of dentate potentials could be induced preferentially during the positive phase of dentate theta in awake and mobile rodents.

As previously mentioned, the septal complex provides cholinergic and GABAergic input to the hippocampus at theta frequency (Brazhnik and Fox 1997) resulting in a continually varying neuromodulatory tone throughout the hippocampus. Given that theta is similar in phase across large portions of the hippocampus (Buzsaki et al. 1990), it is reasonable to infer that this neuromodulatory tone varies similarly throughout the hippocampus as a result of theta input. Therefore this phasic, synchronous neuromodulatory tone may be the cause of the variations in neuronal function that coincide with the theta rhythm.

\section{Theta phasic parameters in awake preparations}

Rao et al. (1998) found a $6 \%$ change in the slope of similar evoked potentials within the CA1 of awake behaving rats exhibiting theta while running on a linear track for food reward. Evoked potential height was measured at the positive and negative peaks of local theta (approximately in phase with dentate theta) and was found to be larger at the positive peak of local theta. This result is correlated with the phasic changes in slope we observed in urethanized rats and is especially interesting in light of the fact that interneurons fire maximally on different phases of dentate theta during urethanized and awake states. Interneuron firing is maximal near the positive peak of dentate theta in the awake rat, but nearer the negative peak in the urethan preparation. It should also be noted that average theta frequencies differ between awake $(7 \mathrm{~Hz}$, $\sim 140$-ms cycle time) and urethan anesthetized states $(3.5 \mathrm{~Hz}$ and $\sim 80$-ms cycle time). This is particularly relevant because processes such as metabotropic receptor activation have onset time courses of tens of milliseconds (Dittman and Regehr 1997; Isaacson et al. 1993). This could mean that substantial variation in frequency results in a substantial change in the phase lag between the maximal release of a substance and its maximal effect. Rudell et al. (1980) found a variation in population spike amplitude in awake rats similar to that in the urethanized preparation but approximately $100^{\circ}$ later in the theta cycle (Rudell and Fox 1984).

\section{Relation to computational modeling}

Despite the differences in the phase of certain effects between urethan and awake preparations, it is clear that the tone 
of neuronal function varies to a significant degree within each theta cycle. These differences could be as distinct as learning versus retrieval modes (Hasselmo 1999) or a more subtle function such as improving the fidelity of sequence recall. Analytical work and simulations by Sohal and Hasselmo (1998a,b) demonstrate how a phasic variation of synaptic strength can help enhance the retrieval of partially overlapping sequences in a model of CA3. The phase with weaker synaptic strength allows afferent input to determine which sequence is recalled, whereas the subsequent increase in synaptic strength enhances retrieval of the full sequence. Wallenstein and Hasselmo (1998) demonstrate how changes in synaptic transmission could result in the phase precession effect exhibited by hippocampal place cells (O'Keefe and Recce 1993) by modulating the length of sequences recalled at different phases of the theta cycle.

\section{Theta versus non-theta effects}

The data here on the size of evoked potential slope during theta and non-theta states contribute to other work that illustrate differences in neuronal function that are correlated with theta and behavioral state in anesthetized and awake preparations (Buzsaki et al. 1981; Herreras et al. 1988a; Leung and Vanderwolf 1980; Núñez et al. 1987; Winson and Abzug 1978). Herreras et al. (1988a,b) have shown that different groups of projections within the hippocampus are differentially modulated by theta relative to non-theta. CA 3 afferents to CA1 are suppressed during theta relative to non-theta states. Perforant path inputs to the dentate gyrus, which could have contaminated these potentials, are enhanced by theta relative to non-theta. The magnitude of the suppression in the Herreras work was not clearly quantified, but examination of the data allowed a rough estimation of the effect size, which appeared to be about $15 \%$. The theta suppression observed here, in conjunction with the Herreras et al. (1988a) result, suggests that the theta-suppressed potentials involved in these experiments were primarily CA3 afferents and not volume conducted dentate potentials, and our observed effect size of $18.2 \%$ agrees with the figures in his paper.

These changes in size of potentials could be directly related to the change in acetylcholine levels observed with microdialysis comparing theta versus non-theta states (Marrosu et al. 1995) and varying degrees of theta amplitude (Monmaur et al. 1997). Acetylcholine has been shown to suppress Schaffercollateral CA1 potentials (Hasselmo and Schnell 1994) and infusion of scopolamine blocks the suppression of CA1 potentials observed by Herreras et al. (1998b) during theta. Leung and Vanderwolf (1980) found that atropine reduces movement induced suppression of Schaffer-CA1 potentials in the awake rat.

\section{O N C L US I O N}

In summary, an effective modulation of the slope of extracellularly recorded excitatory evoked field potentials was found during different phases of theta near the dendrites of hippocampal pyramidal cells. When averaged over the entire data set, evoked potential slope changed by $7.8 \%$, but an average modulation of $9.5 \%$ was observed for individual trial sets within single animals. Evoked potentials were maximal $18^{\circ}$ after the positive peak of dentate gyrus theta and were minimal $198^{\circ}$ after the positive peak. This effect contributes to a wealth of other effects on neuronal function that also varies with the phase of theta rhythm.

The authors thank E. DeRosa, S. Fox, M. Patil, G. Orr, G. Rao, and R. Vertes for advice and comments relating to this work.

This work was supported by National Institute of Mental Health Grant MH-52732 and National Science Foundation Grant IBN9996177.

Address for reprint requests: B. Wyble, Dept. of Psychology, Boston University, 64 Cummington St., Boston, MA 02215.

Received 3 May 1999; accepted in final form 8 December 1999

\section{REFERENCES}

Alonso, A. And Garcia-AustT, E. Neuronal sources of theta rhythm in the entorhinal cortex of the rat. I. Laminar distribution of the theta field potentials. Exp. Brain Res. 67: 493-501, 1987.

Amaral, D. G. AND KuRZ, J. An analysis of the origins of the cholinergic and noncholinergic septal projections to the hippocampal formation of the rat. J. Comp. Neurol. 240: 37-59, 1985.

Bland, B. H. The physiology and pharmacology of hippocampal-formation theta rhythms. Prog. Neurobiol. 26: 1-54, 1986.

BRAZHNIK, E. S. AND Fox, S. E. Intracellular recordings from medial septal neurons during hippocampal theta rhythm. Exp. Brain Res. 114: 442-453, 1997.

Buzsaki, G., Chen, L. S., And Gage, F. H. Spatial organization of the physiological activity in the hippocampal region: relevance to memory formation. Prog. Brain Res. 83: 256-258, 1990.

Buzsaki, G., Grastyan, E., Czopf, J., Kellenyi, L., and Prohaska, O. Changes in neuronal transmission in the rat hippocampus during behavior. Brain Res. 225: 235-247, 1981.

Buzsaki, G., Leung, L. S., And Vanderwolf, C. H. Cellular bases of hippocampal EEG in the behaving rat. Brain Res. Rev. 6: 139-171, 1983.

DitTman, J. S. AND REgeHR, W. G. Mechanism and kinetics of heterosynaptic depression at a cerebellar synapse. J. Neurosci. 17: 9048-9059, 1997.

Fox, S. E. Membrane potential and impedance changes in hippocampal pyramidal cells during theta rhythm. Exp. Brain Res. 77: 283-294, 1989.

Fox, S. E., Wolfson, S., AND RANCK, J. B. J. Hippocampal theta rhythm and the firing of neurons in walking and urethane anesthetized rats. Exp. Brain Res. 62: 495-508, 1986

Freund, T. F. AND BuZsAKI, G. Interneurons of the hippocampus. Hippocampus 6: 347-470, 1996.

Green, E. J., McNaughton, B. L., And Barnes, C. A. Role of the medial septum and hippocampal theta rhythm in exploration-related synaptic efficacy changes in rat fascia dentata. Brain Res. 529: 102-108, 1990.

GREEN, J. D. AND ARduINI, A. Hippocampal electrical activity in arousal. J. Neurophysiol. 17: 533-557, 1954.

Hasselmo, M. E. Septal modulation of hippocampal activity: What is the function of the theta rhythm? In: The Behavioral Neuroscience of the Septal Region, edited by R. Numan. New York: Springer-Verlag, 1999.

Hasselmo, M. E. AND Schnell, E. Laminar selectivity of cholinergic suppression of synaptic transmission in rat hippocampal region CA1: computational modeling and slice physiology. J. Neurosci. 14: 3898-3914, 1994.

Herreras, O., Solis, J. M., Munoz, M. D., Herranz, A. S., Martin del Rio, R., AND LeRma, J. Sensory modulation of hippocampal transmission. I. Opposite effects on CA1 and dentate gyrus synapsis. Brain Res. 461: 290-302, 1988a.

Herreras, O., Solis, J. M., Herranz, A. S., Martin del Rio, R., and Lerma, J. Sensory modulation of hippocampal transmission. II. Evidence for a cholinergic locus of inhibition in the Schaffer-CA1 synapse. Brain Res. 461: 303-313, 1988b.

Hinton, G. E. AND SEJNOwski, T. J. Learning in Boltzmann machines. In: Parallel Distributed Processing: Explorations in the Microstructure of Cognition. Cambridge, MA: MIT Press, 1986, vol. 1, p. 282-317.

Holscher, C., ANwyl, R., AND Rowan, M. J. Stimulation on the positive phase of hippocampal theta rhythm induces long-term potentiation that can be depotentiated by stimulation on the negative phase in area CA1 in vivo. J. Neurosci. 17: 6470-6477, 1997.

HoPfIELD, J. J. AND TANK, D. W. Neural computation of decisions in optimization problems. Biol. Cybern. 52: 141-152, 1985. 
Huerta, P. T. And Lisman, J. E. Bidirectional synaptic plasticity induced by a single burst during cholinergic theta oscillation in CA1 in vitro. Neuron 15: 1053-1063, 1995.

IsAacson, J. S., Solis, J. M, AND Nicoll, R. A. Local and diffuse synaptic actions of GABA in the hippocampus. Neuron 10: 165-175, 1993.

Kamondi, A., Acsady, L., WAng, X. J., And BuzsaKi, G. Theta oscillations in somata and dendrites of hippocampal pyramidal cells in vivo: activitydependent phase-precession of action potentials. Hippocampus 8: 244-261, 1998.

LaCAille, J. C. And Schwartzkroin, P. A. Stratum lacuunosum-moleculare of hippocampal CA1 region. II. Intrasomatic and intradendritic recordings of local circuit synaptic interactions. J. Neurosci. 8: 1411-1420, 1988.

LeunG, L. S. AND VANDERWOLF, C. H. Behavior-dependent evoked potentials in the hippocampal CA1 region of the rat. II. Effect of eserine, atropine, ether and pentobarbital. Brain Res. 198: 119-133, 1980.

LEUNG, L. S. AND YIM, C. Y. Intracellular records of theta rhythm in hippocampal CA1 cells of the rat. Brain Res. 367: 323-327, 1986.

Marrosu, F., Portas, C., Mascia, M. S., Casu, M. A., Fa, M., Giagheddu, M., Imperato, A., AND Gessa, G. L. Microdialysis measurement of cortical and hippocampal acetylcholine release during sleep-wake cycle in the freely moving cats. Brain Res. 671: 329-332, 1995.

Monmaur, P., Collet, A., Puma, C., Frankel-Kohn L., and Sharif, A. Relations between acetylcholine release and electrophysiological characteristics of theta rhythm: a microdialysis study in the urethane-anesthetized rat hippocampus. Brain Res. Bull. 42: 141-146, 1997.

NúÑEZ, A., GARcíA-AustT, E., AND BuÑo, W., JR. Intracellular $\theta$-rhythm generation in identified hippocampal pyramids. Brain Res. 416: 289-300, 1987.

O'KeEFE, J. AND ReCCE, M. L. Phase relationship between hippocampal place units and the EEG theta rhythm. Hippocampus 3: 317-330, 1993.

Orr, G., Rao, G., Stevenson, G. D., Barnes, C. A., and McNaughton, B. L. Hippocampal synaptic plasticity is modulated by the theta rhythm in the fascia dentata of freely behaving rats. Soc. Neurosci. Abstr. 864: $14,1999$.

Paxinos G. And Watson, C. The Rat Brain in Stereotaxic Coordinates. San Diego, CA: Academic Press, 1986.
Rao, G., Orr, G., Barnes, C. A., And McNaughton, B. L. Theta rhythm modulation of CA1 evoked potential size during locomotion. Soc. Neurosci. Abstr. 367: 16, 1998

Rudell, A. P. AND Fox, S. E. Hippocampal excitability related to the phase of the theta rhythm in urethanized rats. Brain Res. 294: 350-353, 1984.

Rudell, A. P., Fox, S. E., AND RANCK, J.B.J. Hippocampal excitability phase-locked to the theta rhythm in walking rats. Exp. Neurol. 68: 87-96, 1980.

Skaggs, W. E., McNaughton, B. L., Wilson, M. A., And Barnes, C. A. Theta phase precession in hippocampal neuronal populations and the compression of temporal sequences. Hippocampus 6: 149-172, 1996.

SoHAl, V. S. AND Hasselmo, M. E. Changes in GABA $_{B}$ modulation during a theta cycle may be analogous to the fall of temperature during annealing. Neural Comp. 10: 869-882, 1998a.

Sohal, V. S. AND Hasselmo, M. E. GABA ${ }_{\mathrm{B}}$ modulation improves sequence disambiguation in computational models of hippocampal region CA3. Hippocampus 8: 171-193, 1998b.

STEWART, M. And Fox, S. E. Do septal neurons pace the hippocampal theta rhythm? Trends Neurosci. 13: 163-168, 1990.

Tsodyks, M. V., Skaggs, W. E., Sejnowski, T. J., And McNaughton, B. L. Population dynamic and theta rhythm phases precession of hippocampal place cell firing: a spiking neuron model. Hippocampus 6: 271-280, 1996.

VERTES, R. P. AND Kocsis, B. Brainstem-diencephalo-septohippocampal systems control the theta rhythm of the hippocampus. Neuroscience 81: 893926, 1997.

Wallenstein, G. V. And Hasselmo, M. E. GABAergic modulation of hippocampal population activity: sequence learning, place field development, and the phase precession effect. J. Neurophysiol. 78: 393-408, 1998

Winson, J. AND ABzUG, C. Neuronal transmission through hippocampal pathways dependent on behavior. J. Neurophysiol. 41: 716-732, 1978.

Wyble, B. P., Linster, C., And Hasselmo, M. E. Evoked synaptic potential size depends on phase of theta rhythm in rat hippocampus. Soc. Neurosci Abstr. 7: 197, 1997. 